\title{
Heterogeneous Networks in 5g using Joint Path Selection and Efficient Self-Organization Protocol

\author{
B.Pavithra, Komala James
}

\begin{abstract}
Tree networks are systematically spread across multiple networks. This project proposes a framework for effective selforganization for $5 \mathrm{G}$ heterogeneous networks. All nodes in this network are classified into two types: network nodes, and nonnetwork nodes. Network nodes are able to broadcast packets to neighboring nodes. A tree-based network can then be obtained from one layer to another. The topology is dynamically balanced to balance energy consumption and extend the service life of the network. To test the current technique we perform tests with it. Results from the simulation show our proposed protocol can easily create a stable tree-based network. As the network size rising, the time for self-organization, average hop and error ratio for packets does not change any more. In comparison, packet success rates in effective self-organization protocol are significantly higher compared to AODV and DSDV. Non-network nodes collect the packets being transmitted and decide whether to access the network. We use various metrics such as number of child nodes, hop, contact distance and residual energy to meet the available sink nodes weight during the self-organizing process, the node with highest weight is selected as sink node. Non-network nodes will be transformed to network nodes if they effectively join the network.
\end{abstract}

Keywords: Self-Organizing Networks (SON), Efficient SelfOrganization Protocol (ESOP).

\section{INTRODUCTION}

\section{GENERAL} running apps that need high data rate demands. One of the approaches to address the data rate constraint for network densification by the installation of small cells. Macro-, Nano-, Pico- and Femto-cells working simultaneously are called heterogeneous networks (HetNets).Heterogeneous networks have been recognized as one of the promising 5G technique to provide wireless backhaul for small cell (SC) deployment. The joint path selection has been employed to improve the downlink transmission by using self-organizing interference management strategy. This has been commonly used in technologies such as localization, factory automation, environmental control and other. Sensor networks consist of a number of randomly dispersed, lowcost , low-power tiny sensor nodes. These nodes will communicate with each other to gather and relay sensing data. With the size that and the upgrading of apps, the network infrastructure is becoming more complex. Network nodes restrict the memory, energy, and computing capability.

Revised Manuscript Received on June 15, 2020.

* Correspondence Author

Miss. Pavithra. B*, Department of Communication Systems from SRM Valliammai Engineering College, Chennai, India. E-mail: kbpavithra1995@gmail.com

Dr. Komala James, Professor, Department of ECE, SRM Valliammai Engineering College, Chennai, India. E-mail: komalj.ece@valliammai.co.in.

(C) The Authors. Published by Blue Eyes Intelligence Engineering and Sciences Publication (BEIESP). This is an open access article under the CC BY-NC-ND license (http://creativecommons.org/licenses/by-nc-nd/4.0/)
Next generation 5 G cellular networks should be

To optimize life, several researchers are engaged in the management of network topology, constructing better data transmission routes and managing node energy consumptions. The idea of Self-Organizing Networks (SON) only took root after the transition from $3 \mathrm{G}$ to $4 \mathrm{G}$ ended. A self-organizing network (SON) is an automated system intended to allow cellular networks preparing, configuring, controlling, improving, and curing.

This is considered a significant requirement for mobile networks and activities largely due to potential cuts in capital spending (CAPEX) and operational expense (OPEX) by the implementation of SON. Because the network is integrated, the operators are now able to focus on delivering an exceptional customer interface.

\section{METHODOLOGY}

\section{CLUSTER BASED FORMATION}

The cluster based formation helps in load balancing but fails to cover a large area because they are one-hop protocols. The tree structures scale well but fail in energy balance. Our main intension is to optimize energy consumption in this self-organizing network and hence prolong network lifetime. The topology discussed above no doubt covers a large area but is prone to energy hole problem if the base station is located far away from the network. A tree-based approach to decisions Is to make full use of decision factors with varying Styles to allow optimum network collection [1]. The energy hole problem is a situation where the data is collected but fails to reach the base station due to energy drain. The solution to this energy hole problem is to introduce mobility in the network. In the proposed system we choose to introduce mobility only to the sink node. The sink node moves freely around the network and collects data from each cluster when it is in the proximity of the cluster head. This collected data is delivered to the base station eliminating energy hole problem. The next problem we address is the energy consumption problem. If frequent construction of routing table, re-clustering, re-election of head, re-sending of data happens, energy drains quickly. To avoid these, we introduce a static agent to reduce the overhead on the cluster head. The static agent takes the responsibility of storing the aggregated data and storing only the overflow of data in the cluster head. It also monitors the mobile sink node 's motion by testing the mobile sink node's lookup table. Energyefficient time synchronization protocol, known as ETSP, which substantially increases the energy consumption over existing time synchronization schemes[2]. 


\section{Heterogeneous Networks in $5 \mathrm{~g}$ using Joint Path Selection and Efficient Self-Organization Protocol}

During the self-organization method, we use different parameters such as the amount of child nodes, hop, contact distance and residual energy to achieve the weight of the usable sink nodes, the maximum weight node chosen as sink node [3].

This frees the cluster head of main responsibilities reducing energy consumption. We just don't need to minimize total energy consumption but also keep WSN in balance loading[4]. For cluster-based routing protocols, three primary strategies are selection of cluster heads, cluster formation and data transmission[5]. Optimize the existence of wireless sensor networks by picking the farthest cluster head instead of the nearer cluster head and creating a cluster using the following parameters, such as residual capacity, intracluster distance and node density [6].

The proposed system model has the following assumption:

1. Wireless sensor nodes are randomly deployed in a twodimensional area.

2. There is only one mobile sink node deployed in the area which has random motion with infinite energy.

3. The deployed sensor nodes are stationary and also energy constrained. These will operate as long as the energy is not exhausted.

4. Sensor nodes are location-aware by using the position (co-ordinates) algorithms used in the system and are updated in the lookup table.

The proposed system can be divided into following phases:

1. Step of constructing a tree

2. Step in data processing

3. Step of data transmission

\section{a. Step of constructing a tree:}

Whenever network has data to send, it begins with the initialization. The network is divided into the defined number of clusters roughly based on the selection of area. The node with the maximum residual energy is designated as the center of the cluster. The Head of the cluster transmits its position and remaining resources to all network nodes. All the nodes in the network broadcast their information such as residual energy, distance, and hops to its neighboring nodes. Each network node measures its fitness for the next cluster head using certain network parameters[7]. These are recorded in the table maintained by each node. Each node is hence aware of the location of its neighboring nodes. By looking into this table it decides whether neighboring node should become its parent or child node. If the neighboring node has more control than the opposing node would become the node's parent. If the neighboring node has less energy, it becomes their child node. If there are more than one node having same residual energy the tie is broken by using the distance, hops and the number of children node they possess. This builds a tree structure with the cluster head as the root node. Each node registers itself with the cluster head by providing the metrics. Therefore the head of the cluster is aware of every node in the cluster. Each of the nodes is automatically selected to be the static handler.

\section{b. Step in data processing:}

The child node detects the data and transmits it to the parent node instantly. For each child node the parent node gathers data in a designated location. At last, the parent node aggregates all the data obtained from the kids node. The parent node takes this knowledge up to the next higher level. Maximize network lifespan by optimizing the balance of energy loads for both homogeneous and heterogeneous sensor networks[8]. The cycle persists until it hits the end of the cluster. And the head of the cluster already has all of the aggregated data from both of the kids node.

\section{c. Step of data transmission:}

The aggregated data is stored in the static agent, reducing the storage overhead on the cluster head. The static agent tracks the movement of the mobile sink node. It's updated about the mobile sink node location from the mobile sink node's lookup chart. The static agent moves the accumulated aggregated data to the mobile sink node while the mobile sink node is going inside the vicinity of the cluster. Specific cluster creation and head selection strategies helping to conserve resources [9].

In the following two cases the network is re-constructed partially to balance the energy.

\section{The cluster head drains energy below a threshold}

If the cluster head energy falls below a threshold (say R\%), You must remove the farthest child link. R percent is focused on the shift of last topology's residual energy. This involves removing the entry of the child node in the child table of the cluster head and informing the child node to re-elect the best cluster head based on the decided metrics. Replacing the cluster head by ensuring cluster size is not too large or too small[10]. This controls the resources within the cluster and increases the network 's lifespan.

\section{Link failure}

Periodic data packets are transmitted between the head of the cluster and the child node to insure that relations are formed. Self-organizing multihop clustering protocol with versatile initialization time that makes it suitable for wide-size wireless sensor networks[12]. Autonomous multihop clustering protocol that has a flexible initialization time making it ideal for wireless sensor networks of wide size[13]. If the cluster head does not receive any packets from the child, This concludes that the infant is dead, and prevents the infant table entry. If the child gets no comment from the head of the cluster, instead a new cluster is reelected.

\section{RESULTS AND DISCUSSION}

We used MATLAB to simulate this to test our proposed model. Use of a cluster-based self-organizing approach to manage the femtocell spectrum and power resources[14]. The new Guideline on Successful SelfOrganization has three functions:

1. Tree-based network self-organize,

2. Equilibrium energy utilization,

3. Reelect node on the drain.

\section{Published By:}

Blue Eyes Intelligence Engineering

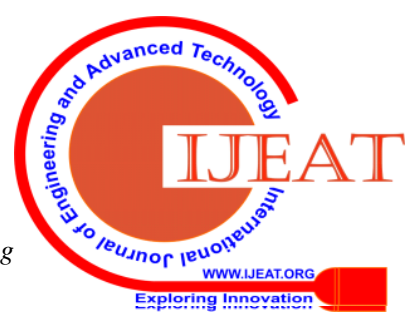


We need to set parameters before establishing a tree-based network. The average radius of contact is $15 \mathrm{~m}$, hence is $15 \mathrm{~m}$. The full number of nodes for the infant is 10 so that is 11 . The average init-energy is $29 \mathrm{~J}$ so is $1 / 29$. There's a full hop of 10 and is 11.100 sensor nodes are spread uniformly over the $100 \mathrm{~m} * 100 \mathrm{~m}$ region in the simulation.

Test cases for energy

1. When energy of cluster head $<$ threshold.

2. When energy of cluster head $=0$.

3. When node/child energy $<$ threshold.

4. When node/child energy $=0$.

5. When static agent energy drains or less than threshold.

In the data convergence, data sensed by the child node will be sent to their respective parent node until it converges at cluster head. An sufficient and efficient selection of cluster-heads during cluster forming[11]. A random static agent is chosen to track mobile sink node is in proximity, it should send message to cluster head to transfer data. Utilizing a cluster-based self-organizing strategy to femtocell spectrum and power capital management[15].

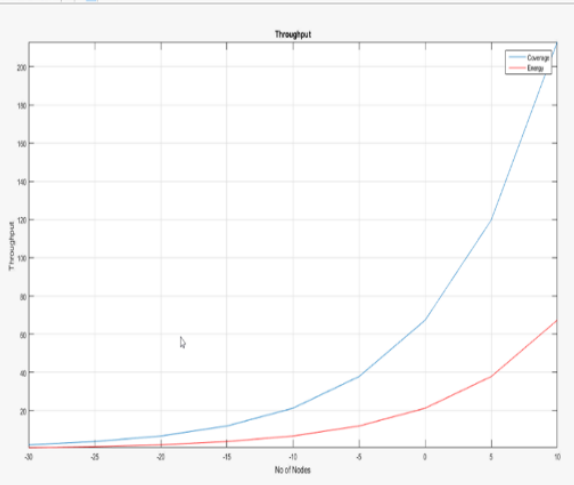

Fig 1. No. of nodes vs throughput

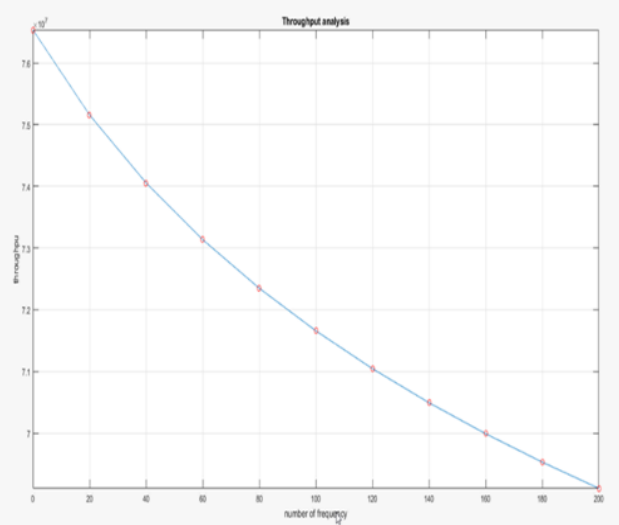

Fig 2. Throughput Analysis

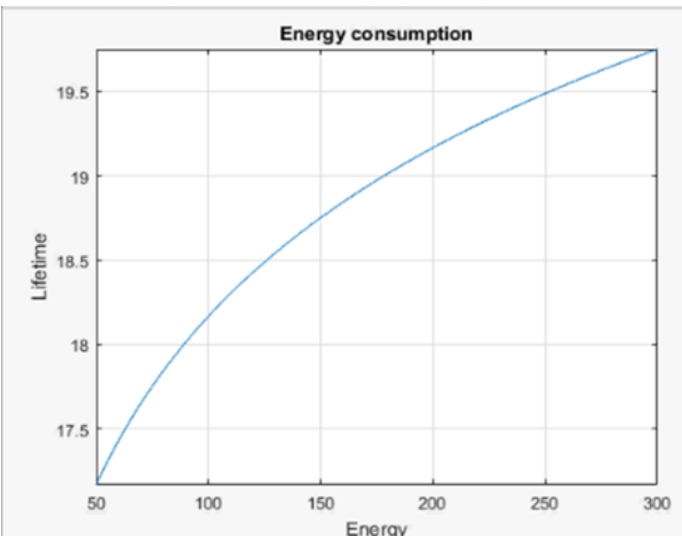

Fig 3. Energy consumption

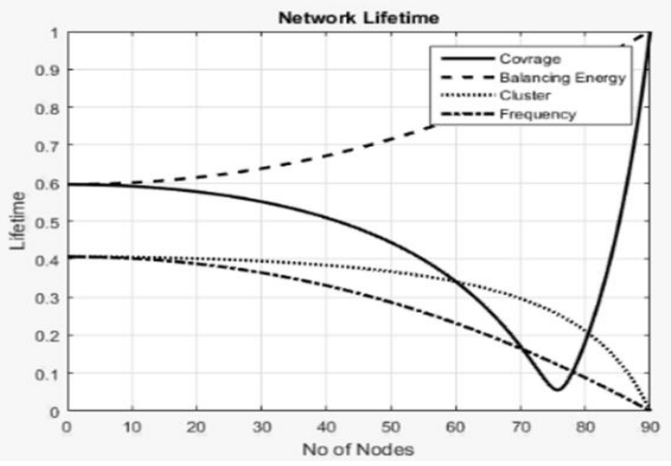

Fig 4. Network Lifetime

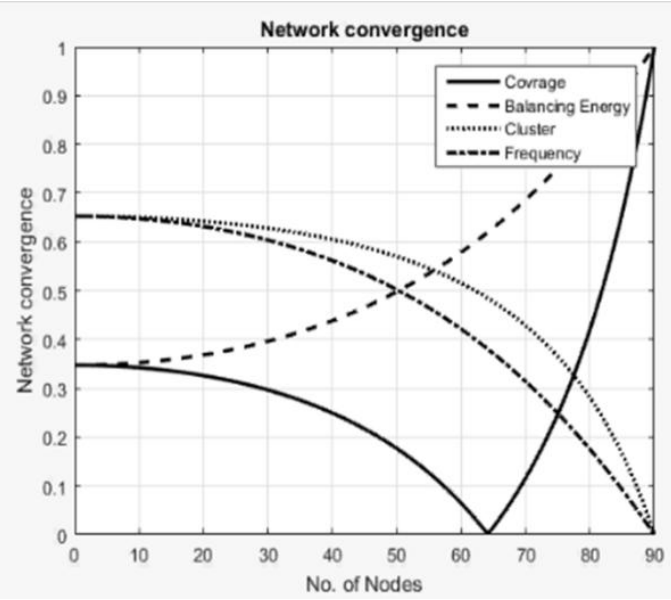

Fig 5. Network Convergence

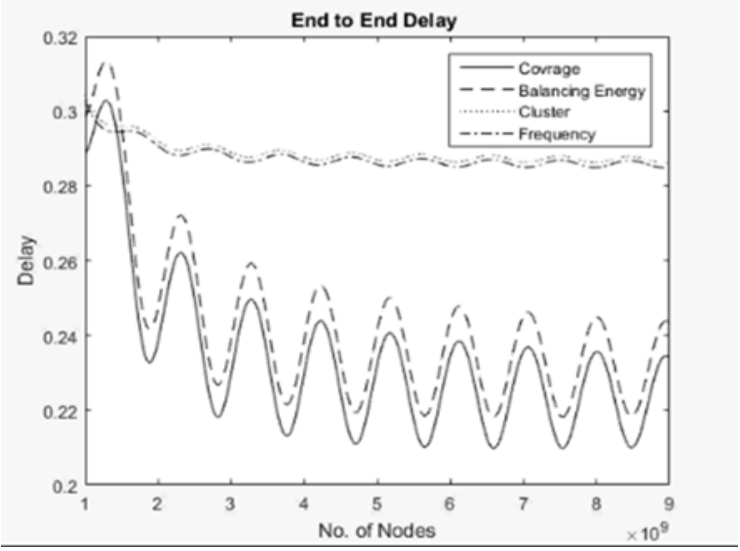

Fig 6. End to End Delay

Published By:

Blue Eyes Intelligence Engineering

Retrieval Number: E1017069520/2020CBEIESP

DOI: 10.35940/ijeat.E1017.069520

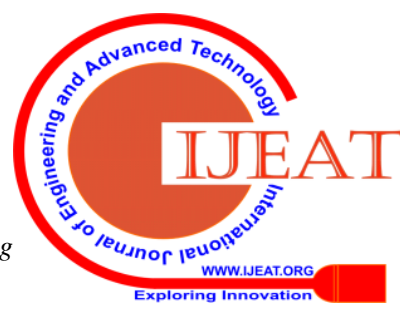




\section{Heterogeneous Networks in $5 \mathrm{~g}$ using Joint Path Selection and Efficient Self-Organization Protocol}

\section{CONCLUSION}

We suggest an effective self-organization protocol that saves more resources by easily creating a tree-based network and has a longer network lifespan. We use the weight of nodes to decide if the node may be a sink node, including residual energy, jump, number of child nodes and gap between the nodes. And the tree depth is balanced for this protocol. During the data transmission cycle the topology of the network dynamically shifts. Due to the energy consumption of sink nodes each sink node would be dynamically reselected is faster than other nodes.

The simulation results demonstrate that the joint path selection and efficient self-organization protocol are capable of creating robust tree-based networks that minimize energy consumption and increase sensor networks' lifespan.

\section{REFERENCES}

1. Y. Wang and K. Zhang, "Decision tree based unsupervised learning to network selection in heterogeneous wireless networks," IEEE Consumer Communications and Networking Conference (CCNC), pp. 1108-1109, 2011.

2. K. Shahzad, A. Ali and N. D. Gohar, "ETSP: An Energy-Efficient Time Synchronization Protocol for Wireless Sensor Networks," 22nd International Conference on Advanced Information Networking and Applications -Workshops, pp. 971-976, 2008.

3. T. Qiu, X. Liu, L. Feng, Y. Zhou and K. Zheng, "An Efficient TreeBased Self-Organizing Protocol for Internet of Things," in IEEE Access, vol. 4, pp. 3535-3546, 2016.

4. Z. Han, J. Wu, J. Zhang, L. Liu and K. Tian, "A General SelfOrganization Tree-Based Energy-Balance routing protocol for wireless sensor network," 18th IEEE-NPSS Real Time Conference, pp. 1-6, 2012.

5. Changjiang Jiang, Min Xiang and Weiren Shi, "Overview of clusterbased routing protocols in wireless sensor networks," International Conference on Electric Information and Control Engineering, pp. 3414-3417, 2011.

6. M. K. Praveen and T. Senthil, "Lifetime maximization of wireless sensor networks using energy-efficient cluster formation strategy," 2014 IEEE International Conference on Computational Intelligence and Computing Research, pp. 1-5, 2014.

7. Nagesha and S. S. Manvi, "A Fuzzy based Approach to Cluster Formation in Wireless Sensor Networks to Enhance the Lifetime of the Network," 2018 3rd IEEE International Conference on Recent Trends in Electronics, Information \& Communication Technology (RTEICT), pp. 1191-1195, 2018.

8. S. A. Ali, C. Sevgi and A. Kocyigit, "Balancing Energy Loads in Wireless Sensor Networks through Uniformly Quantized Energy Levels-Based Clustering," 2010 Fourth International Conference on Sensor Technologies and Applications, pp. 19-23, 2010.

9. S. Lavanya and S. Prakasam, "Performance analysis of cluster formation schemes for energy conservation in wireless sensor networks," 2017 International Conference on Algorithms, Methodology, Models and Applications in Emerging Technologies (ICAMMAET), pp. 1-5, 2017.

10. S. Islam, M. J. Akhtar, M. N. I. Khan and S. J. Islam, "Energy Balanced Clustering using Balancing Nodes for Wireless Sensor Networks," 2019 International Conference on Computer Communication and Informatics (ICCCI), pp. 1-4, 2019.

11. F. D. Tolba, W. Ajib and A. Obaid, "Distributed clustering algorithm for mobile wireless sensors networks," SENSORS, 2013 IEEE, pp. 14, 2013.

12. R. Misra and C. Mandal, "Self-Healing for Self-Organizing Cluster Sensor Networks," Annual IEEE India Conference, pp. 1-6, 2006.

13. O. Saglam and M. E. Dalkili, "A Self Organizing Multihop Clustering Protocol for Wireless Sensor Networks," Fifth International Conference on Mobile Ad-hoc and Sensor Networks, pp. 33-40, 2009.

14. H. M. N. Bandara, A. P. Jayasumana and T. H. Illangasekare, "Cluster Tree Based Self Organization of Virtual Sensor Networks,“ IEEE Globecom Workshops, pp. 1-6, 2008.

15. W. Lai and T. Lee, "A Distributed Cluster-Based Self-Organizing Approach to Resource Allocation in Femtocell Networks," IEEE 75th Vehicular Technology Conference (VTC Spring), pp.1-5, 2012.

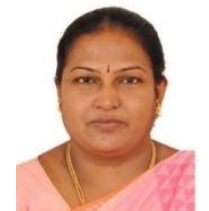

\section{ABOUT THE AUTHORS}

Miss Pavithra. B pursuing her M.E in Communication Systems from SRM Valliammai Engineering College, Chennai, India. She completed B.E (Electronics and Communication Engineering) in Sudharsan Engineering College, Sathyamangalam, Pudukkottai, India. Her area of interest is Optical Communication, Telecommunication Technology. Email: kbpavithra1995@gmail.com.

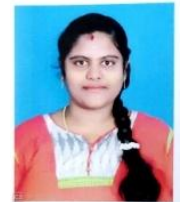

Dr.Komala James is PROFESSOR \& HEAD of the Dept of ECE at SRM Valliammai Engineering College. She has done her B.E in ECE, M.E in Applied Electronics and $\mathrm{PhD}$ in "Mobility issues in Fourth Generation Wireless Networks". She is a FELLOW of IETE, LIFE MEMBER of ISTE \& CSI. Her areas of interest are Heterogeneous Networks, 4G \& 5G Networks, Wireless Communication. Email: komalj.ece@valliammai.co.in
Published By: Blue Eyes Intelligence Engineering

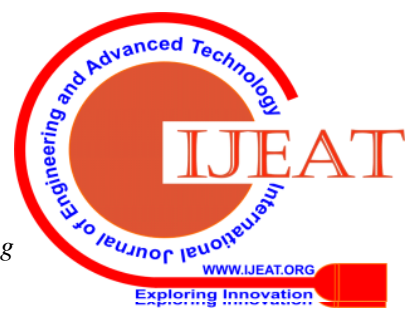

